\title{
Experiencia docente en la adecuación de prácticas de laboratorio presenciales a modalidad virtual
}

\begin{abstract}
Marta Caballero-Jorna ${ }^{a}$, Ester Giménez Carbób y Pedro Serna ${ }^{a}$
a Universitat Politècnica de València, Instituto de Ciencia y Tecnología del Hormigón, Valencia (España), marcajor@upv.es, ${ }^{\mathrm{b}}$ Universitat Politècnica de València, Departamento de Ingeniería de Construcción y Proyectos de Ingeniería Civil, Valencia (España), esgimen@cst.upv.es y ${ }^{a}$ Universitat Politècnica de València, Instituto de Ciencia y Tecnología del Hormigón, Valencia (España), pserna@cst.upv.es.
\end{abstract}

\section{\$EWWFW}

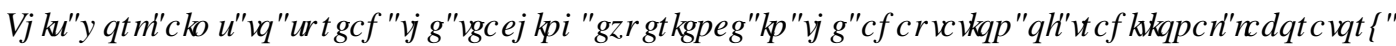

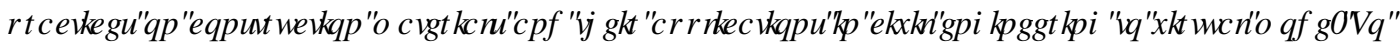

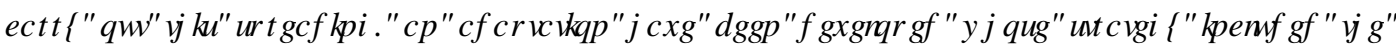

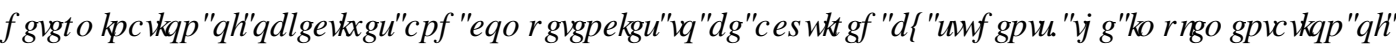

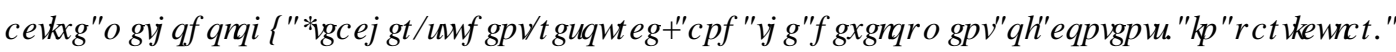

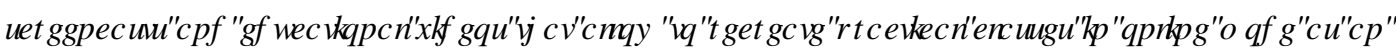

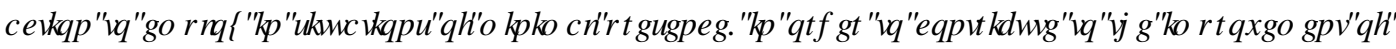

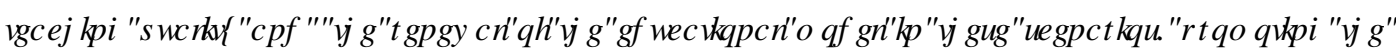

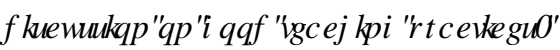

प

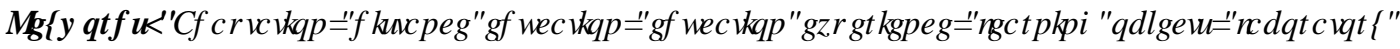
SUFWAHW

\section{HXP HQ}

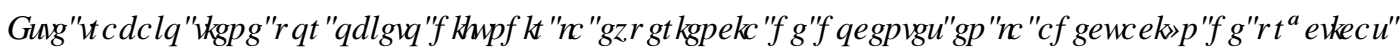

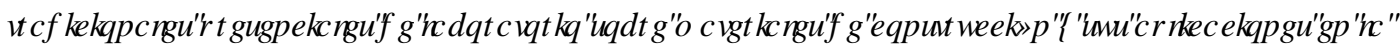

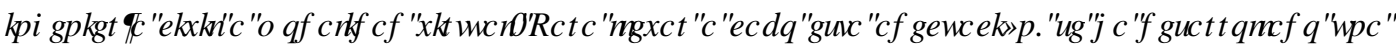

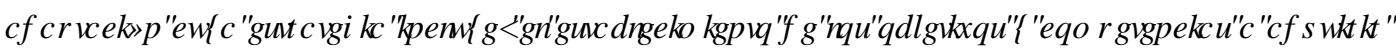

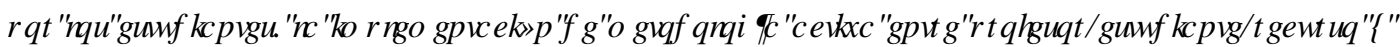





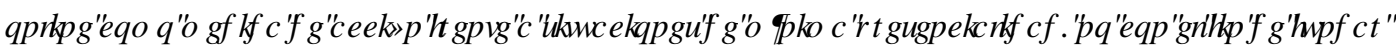

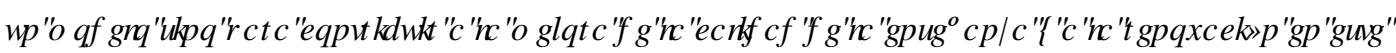

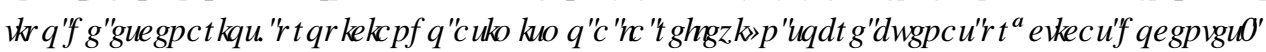
प

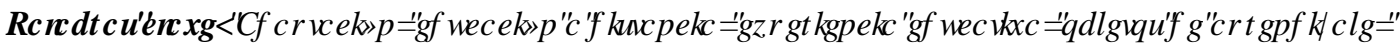
SU FUFDVGHCERLDURURT 


\section{Introducción}

La crisis sanitaria derivada de la COVID-19 ha provocado un cambio abrupto en el contexto educativo dentro del sistema universtario español (SUE), dado que la situación actual ha obligado a la transición y adecuación de la modalidad de enseñanza presencial a la modalidad no presencial (García-Planas y Taberna Torres, 2020), con el objetivo de adaptarse a las constantes modificaciones que se han producido y que aún no han cesado, para poder garantizar la continuidad en la educación sin que ello suponga minorar los estándares de calidad de la enseñanza impartida. Actualmente, el SUE está formado 84 universidades, de las que 50 son públicas, 32 son privadas y 2 son especiales. En relación con la presencialidad, 74 universidades son presenciales y 6 no, excluyendo a las universidades especiales (García-Peñalvo y Rodríguez-Conde, 2020).

A pesar de su estructura organizativa, la mayor parte de universidades presenciales compagina sus procedimientos tradicionales con oferta no presencial. Sin embargo, el modelo educativo no presencial que se ha ido implementando en la formación universitaria tradicional en las últimas décadas, con independencia de si estas son públicas o privadas, es limitado comparado con las universidades creadas específicamente para desarrollarse en el ámbito virtual. Durante el último año, se ha evidenciado que la adopción de modelos de formación online en universidades presenciales dentro del SUE no ha supuesto un problema tecnológico (García-Peñalvo y Rodríguez-Conde, 2020). Las universidades presenciales, entre las que se encuentra la Universitat Politècnica de València (UPV), han dado respuesta a la coyuntura actual, la cual ha planteado nuevos retos docentes y ha fomentado nuevas oportunidades de desarrollo en innovación educativa.

A este respecto, según los últimos comunicados de la Conselleria de Innovación, Universidades, Ciencia y Sociedad Digital y del Vicerrectorado de Estudios, Calidad y Acreditación, con relación al acuerdo del Sistema Universitario Público Valenciano, la Junta de Centro de la Escuela Técnica Superior de Ingeniería de Caminos, Canales y Puertos (ETSICCP) acordó la adaptación extraordinaria al modo de mínima presencialidad en todas sus titulaciones para el segundo cuatrimestre del curso 2020/2021. Con el fin de hacer frente a esta situación y estar en línea con el plan estratégico institucional, se han usado las Tecnologías de la Información y la Comunicación (TICs), como herramienta pedagógica-didácticaformativa, para adaptar las prácticas de laboratorio tradicionales sobre materiales de construcción y sus aplicaciones en la ingeniería civil a la modalidad online.

En este contexto, la percepción de los estudiantes sobre la calidad y ventajas de los entornos virtuales en su aprendizaje y en el desarrollo de competencias y habilidades, el acceso a la plataforma, programas, cursos y la disponibilidad y uso de los diversos recursos tecnológicos y condiciones que las universidades y docentes les facilitan son las variables más destacadas (Casal, 2014; Martínez Rodríguez y González Martínez, 2018; Vite Cevallos, 2020). La creación de contenidos y/o material para su publicación como recurso didáctico tiene una alta relevancia también en la educación virtual para ellos y, concretamente, la innovación planteada en el presente trabajo está basada en la construcción de objetos de aprendizajes (OA) para reproducir las prácticas de laboratorios presenciales.

Sin embargo, estos contenidos por sí solos (vídeos didácticos y screencasts) no garantizan la calidad del proceso formativo. Es importante que el docente promueva el desarrollo de competencias, de manera que garantice el aprendizaje continuo y autónomo, y asimismo, facilite la comunicación en el curso siendo motivador de actividades que apoyan la autorregulación del aprendizaje y el tiempo por el propio alumno (Castillo et al., 2017). A este respecto, las palabras que resultaron como resultado de un estudio para identificar las características de un buen profesor en modalidad en línea por parte del alumnado fueron responsable, accesible y disponible (Bazán-Ramírez, A. HWDQ, 2020), por lo que, apoyándonos en la 
literatura existente, se han implementado en el presente trabajo, metodologías activas entre profesorestudiante-recurso.

En conclusión, esta transformación digital ha supuesto un reto para el equipo docente, no tanto desde la adquisición de nuevas habilidades instrumentales (tecnología, lenguaje, etc.) y/o nuevos conocimientos (formación del profesorado), sino más bien desde la toma de decisiones relacionadas con las estrategias a seguir, desde el diseño pedagógico a las metodologías docentes a emplear.

\section{Objetivos}

El objetivo principal del trabajo es el de presentar y dar a conocer la experiencia educativa docente en el proceso de adaptación de clases prácticas de laboratorio presenciales que tienen lugar en las clases de la asignatura "Materiales de Construcción y sus aplicaciones a la Ingeniería Civil" del Grado en Ingeniería de Obras Públicas (GIOP) y en el Grado de Ingeniería Civil (GIC) de la Universitat Politècnica de València (UPV), a la docencia no presencial, como consecuencia de la situación derivada de la evolución de la COVID-19. La motivación para llevar a cabo este trabajo ha sido la búsqueda de alternativas para salvaguardar al máximo la docencia ya planificada en ambos casos, poniendo al alcance del estudiante los recursos necesarios en su proceso de aprendizaje, sin que ello supusiera una merma en la calidad de la educación impartida.

Para la consecución del objetivo principal de este trabajo, se han establecido una serie de objetivos específicos, que están detallados a continuación:

- Contribuir a la mejora continua y renovación del modelo educativo en situaciones extraordinarias de mínima presencialidad.

- Crear objetos de aprendizaje como material educativo para su uso en la docencia virtual, de modo que se aseguren unos contenidos actualizados y se amplíe el acervo audiovisual institucional.

- Implementar metodologías activas que favorezcan un aprendizaje activo, autónomo, significativo y colaborativo, para minimizar los efectos negativos que pudieran generarse debidos al cese de esta presencialidad.

\section{Desarrollo de la innovación}

Para afrontar el desafío de la adaptación de la modalidad no presencial de las prácticas, se ha seguido la estrategia representada en la Figura 1:

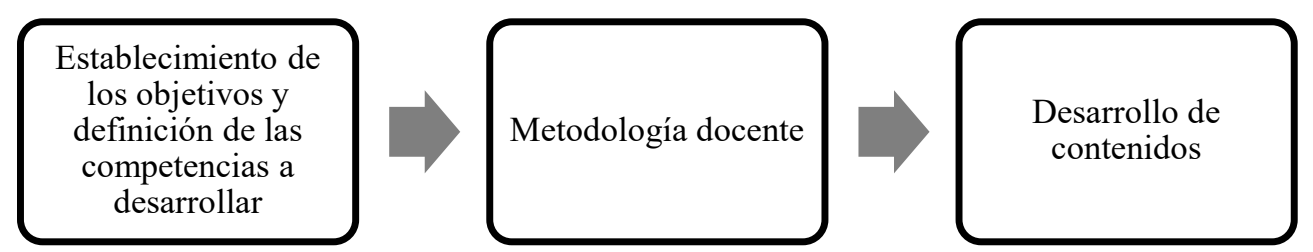

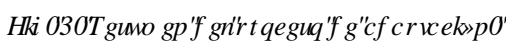

El proceso de adaptación se ha diseñado con el objetivo prioritario de facilitar la docencia y el aprendizaje por medio de la interacción con los materiales didácticos (de modo que los alumnos tengan la posibilidad de ser expuestos a situaciones similares a la de las prácticas de laboratorio) y la interacción entre los distintos miembros implicados en el proceso educativo. 
Las decisiones tomadas en este proceso de adaptación junto con la temporalización y la evaluación son el resultado de reflexionar sobre el papel del profesor para preservar de los estándares de calidad académica en clases tipo prácticas de laboratorio. El hecho de transformar estas prácticas tradicionales a online supone más que integrar y enseñar contenidos que resulten solo expositivos, esto es, no se pueden ejemplificar los temas solamente con el discurso y demostración. La adaptación debe proporcionar capacidad de deducción, compresión, experimentación de los estudiantes y reflejar la utilidad dentro de su ámbito profesional inminente y en su integración con otras disciplinas, respaldado por la atención y disponibilidad del profesorado.

Teniendo en cuenta esto, la metodología docente que se ha adoptado está basada en la propuesta por Cotano (Cotano, 2005), en la que se potencia el rol del profesor como diseñador de medios, facilitador de aprendizaje y orientador del estudiante. Con respecto al modelo pedagógico, la interacción es muy alta, facilitando la flexibilidad al acceso del material y permitiendo el seguimiento de los estudiantes, estableciendo tiempos de respuesta para que los estudiantes puedan recibir la realimentación necesaria sin que esto les impida seguir el desarrollo sus objetivos y competencias.

En este caso, la definición de objetivos y competencias se han mantenido conforme a los previamente planificados en modalidad presencial para ambos grados, GIOP y GIC. La asignatura "Materiales de Construcción y sus aplicaciones a la Ingeniería Civil" es semestral, de carácter obligatorio y se imparte en el segundo curso en cada uno de los grados mencionados. El tamaño de grupo es pequeño (menor de 20 alumnos), por tratarse de clase prácticas de laboratorio y desde el inicio de su implementación en ambos grados en la UPV, se ha desarrollado de forma presencial (teniendo en cuenta su idiosincrasia).

En relación con la preparación de contenidos multimedia, los servicios universitarios no han sido fundados para ello, pero se han utilizado para dar cobertura a la casuística presente. La producción del material se ha llevado a cabo con los medios de grabación del Departamento de Ingeniería de la Construcción y de Proyectos de Ingeniería Civil (tanto medios materiales: cámara de vídeo, trípode, cámara de fotografía, etc. como humanos: técnicos de laboratorio y profesorado de la unidad docente). Las grabaciones han tenido lugar en los laboratorios de prácticas docentes de materiales de construcción del Edificio 4F de la UPV. Se han desarrollado OA para dar respuesta al aprendizaje del estudiante de un procedimiento concreto (Figura 2), en este caso, los vídeos didácticos tienen la finalidad de mostrar el desarrollo de ensayos en materiales de construcción (contenidos procedimentales), considerando el hándicap de la no reproducibilidad por parte del alumnado y los screencast, por su parte, tienen el objetivo de explicar contenidos conceptuales.

Estos OA son de autoría del profesorado de la unidad docente, tienen la duración recomendada de entre 3 y 10 minutos y permiten el aprendizaje guiado pero autónomo del estudiante. Los screencasts y vídeos didácticos son claramente asíncronos permitiendo su orientación a la reutilización en cualquier momento. La principal diferencia que presentan entre ellos es que, en los screencasts, se muestran los contenidos generados mediante capturas de la pantalla, acompañados de la voz en off del profesor locutor y, por el contrario, en los vídeos didácticos, se muestran videos en movimiento montados a partir de filmaciones realizadas con una cámara de video y su posterior edición, con la correspondiente grabación de sonido en directo. La herramienta empleada para la edición de vídeos ha sido Adobe Presenter, muy utilizada en el Flipped Learning (González y Serrano, 2017). 

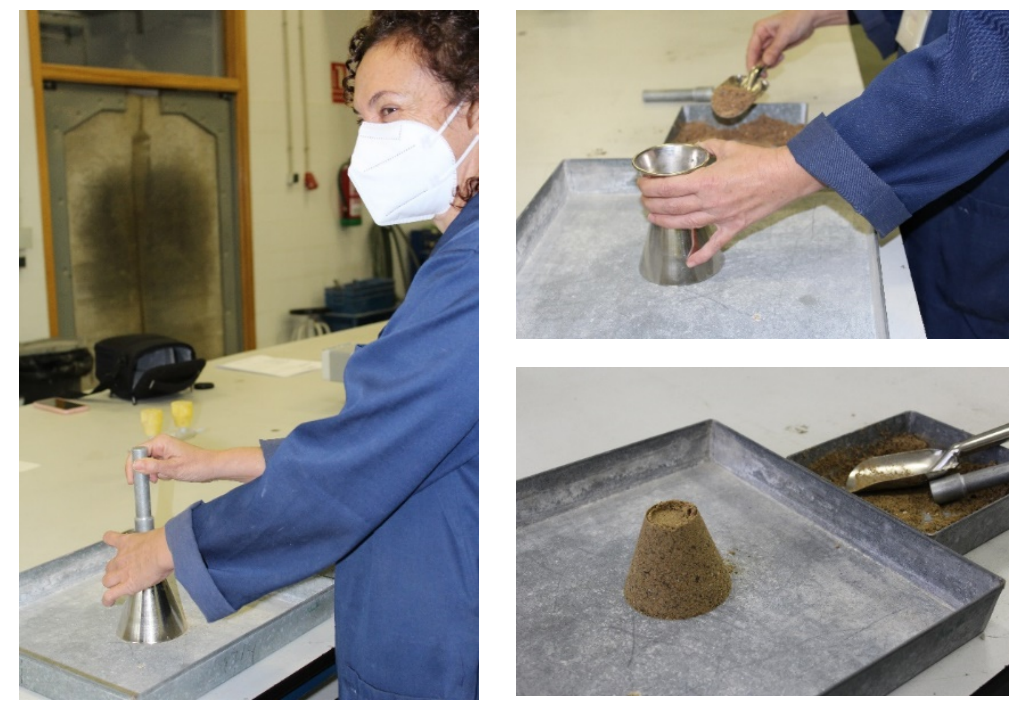



La presente adaptación incluye la adecuación de cuatro prácticas presenciales de laboratorio a modalidad online, a través de la grabación y edición de los citados OA.

Para la primera práctica, se prepara material que los alumnos deben entregar a través de INTRANET (Sistema de Gestión de Aprendizaje coorporativo), basado en resolución de problemas y un screencast con generalidades de materiales de los materiales, es decir, sobre sus propiedades, planteando una pregunta final para dar mayor dinamismo y hacer el video interactivo.

Para la segunda práctica, se graban dos vídeos didácticos con dos ensayos, conocidos como "Ensayos para determinar las propiedades geométricas de los áridos. Parte 8: Evaluación de los finos. Ensayo del equivalente de arena" y "Ensayos para determinar las propiedades geométricas de los áridos. Parte 9: Evaluación de los finos. Ensayo de azul de metileno." con el fin de ejemplificar la realización de los procedimientos. La demostración de estos tests que se realizan frecuentemente en el ámbito de la construcción, la explicación de su fundamento y la interpretación de sus resultados proporcionan herramientas que capacitan al alumnado para la adquisición de competencias transversales (toma de decisiones); se enfoca el contenido desde un punto de vista actitudinal, esto es, el alumno tiene que analizar, tras ver el video, la aptitud que tiene un determinado árido para diferentes aplicaciones dadas.

Para la tercera práctica, el contenido del screencast incluye la explicación de conceptos relacionados con cemento, tal como, pasta de consistencia normal y varios fragmentos de video en los que se realiza la búsqueda de la relación agua/cemento para determinar la pasta de consistencia normal. También, aparece la fabricación de probetas de mortero de cemento normalizado y hormigón.

Para la cuarta y última práctica, los alumnos tuvieron que determinar las características de una barra corrugada (previamente identificada) a partir de un OA en el que se recoge un ensayo de resistencia a tracción de dicha barra. Adicionalmente, se realizó un montaje de fragmentos de videos con las diferencias conceptuales (en el diagrama tensión-deformación) entre los comportamientos frente a roturas de los distintos materiales con los que se ha trabajado en las prácticas anteriores.

Todo este material educativo está alojado en la INTRANET, plataforma a través de la cual se hace entrega del material virtual. En referencia a la interacción con estos contenidos, el método de los estudiantes va desde la actividad hacia el contenido, al contrario de la práctica habitual que va desde la exposición temática hasta los ejemplos y actividades. 
En este caso, los OA toman la mayor representatividad en la adaptación, más que los contenidos textuales. Además de esto, las actividades que estaban planificadas para realizarse se han convertido en actividades online (a través de dosier entregables), utilizando INTRANET. Se utilizaban también los equipos instalados en las aulas para la emisión síncrona de sesiones de resolución de dudas o teóricas correspondientes a estas prácticas (vía Microsoft Teams); se prefirió esta fórmula de educación a distancia para ese tipo de sesiones por su similitud con la dinámica tradicional y por ser la fórmula más valoradas por los estudiantes (UNESCO, 2020). Estas sesiones fueron combinadas con tutorías individuales para resolver con más detalle las dudas conceptuales, dudas en los ejercicios prácticos, etc. prestando atención a la interacción con los estudiantes, dado que es fundamental para que se produzca aprendizaje, que haya colaboración y participación activa por parte de todos los involucrados en la acción formativa.

\section{Resultados}

El proceso de adaptación propuesto está actualmente implementado. Con su puesta en marcha, se da a conocer parte del panorama educativo actual derivado de la COVID-19 en la docencia universitaria, así como permite enfatizar la importancia de la pedagogía para alcanzar una enseñanza de calidad en la modalidad online, permitiendo identificar qué prácticas docentes favorecen el e-Learning. Entre ellas, destacan desde el punto de vista del docente, la mayor comunicación y coordinación entre los distintos agentes de la acción formativa.

En esta adaptación, se han empleado las TICs. La estrategia desarrollada ha permitido fortalecer las destrezas y habilidades de los docentes en el uso de plataformas virtuales, recursos tecnológicos, etc., favoreciendo igualmente a la interacción docente-estudiante. Asimismo, es importante destacar en relación a perspectiva docente que, en general, el tiempo de dedicación a la docencia aumentó para la creación contenido virtual (preparación de los $\mathrm{OA}$ ) y para la adaptación a modalidad no presencial comparado con las clases planificadas en origen para su presentación presencial.

Además de esto, es necesario crear cronogramas y planificar metas alcanzables para el alumnado, para orientar al estudiante en su proceso de aprendizaje y permitir que alcancen los objetivos previstos y las competencias genéricas y multidisciplinares (como, por ejemplo: la gestión de la información, habilidades informáticas básicas y trabajar de forma autónoma).

Aún es pronto aún para extraer conclusiones, extrapolar resultados y hacer una autocrítica sobre la adaptación, quedando pendiente y siendo esta parte del estudio esencial para la posterior definición de los aspectos no adecuados y mejorar la experiencia adaptativa. Así mismo, se espera, en futuros trabajos, conocer las percepciones de los estudiantes sobre sus vivencias formativas de esta adaptación de prácticas de laboratorio en modalidad virtual a través de una retroalimentación de los mismo (podría ser mediante encuestas) y a través de la evaluación del método empleado, analizando los resultados del proceso de aprendizaje del alumnado.

\section{Conclusiones}

La asignatura "Materiales de Construcción y sus aplicaciones a la Ingeniería Civil" se ha impartido presencialmente desde el inicio de la implementación de los grados de GIOP y GIC en la UPV. La situación dada por la COVID-19 ha motivado adoptar nuevas metodologías dentro de la asignatura en ambos grados, así como el desarrollo de nuevos contenidos, eliminando los prejuicios sobre la no presencialidad en el aprendizaje.

La experiencia compartida aquí presenta las acciones desarrolladas para adecuar prácticas de laboratorio a la educación online y para hacer frente a futuras situaciones de no presencialidad que puedan darse en el contexto académico. De esto, se puede concluir que es posible diseñar y adaptar la parte práctica de 
laboratorio de modalidad presencial a online y para tal fin, desde el punto de vista docente, los objetos de aprendizaje producidos han demostrado ser herramientas muy útiles junto con el empleo de metodologías activas.

Es cierto que el aprendizaje en las prácticas presenciales involucra tanto la aplicación de los conocimientos adquiridos durante las clases teóricas como la experimentación y demostración, y también es importante destacar que las clases tipo laboratorio presencial tienen características que resultan difíciles de reproducir debido a su idiosincrasia, la cual difiere mucho de la de las clases teóricas o aquellas pensadas para desarrollarse en un contexto plenamente online, pero se ha diseñado esta parte de la asignatura de modo que los alumnos tuvieran demostraciones metodológicas a través de los OA. Sin embargo, la evaluación de esta adaptación por parte de estos últimos se realizará en futuros trabajos, dada la reciente implementación de la misma.

En resumen, con este trabajo se incrementa el conjunto de vivencias del profesorado como participantes en los procesos de enseñanza-aprendizaje online con el propósito de colaborar en la mejora continua de la calidad del mismo y se deriva que es posible realizar la adecuación de la parte práctica de laboratorio de modalidad presencial a online, mediante la ejemplificación de este caso concreto, compartiendo la experiencia para otros docentes que quieran adaptar las prácticas de laboratorio presencial a modalidad virtual, con el empleo las TICs.

\section{Referencias}

\section{Libros}

GARCÍA-PEÑALVO, F.J. Y RODRÍGUEZ-CONDE, M.J. (2020) 3DQRU P IFD GHDHV HDQQQ $\square$ HQ HD UWHP DXQIYHWWURIHSD RQRMadrid, España: Editorial Bubok.

\section{Artículo de una revista}

BAZÁN-RAMÍREZ, A. ET AL. (2020). “Accesibilidad, dificultades y ventajas del estudio online por COVID-19 en un posgrado presencial en educación" en 3 LRSy ULRVI 55 HLUMQWFIRQH, 8(3), p. e659.

CASAL, M. S. (2014). “Metodología Didáctica En Entornos Virtuales De Aprendizaje” en ( WFDQHypp. 19.

CASTILlO, M. A. S. ( 7ロ\$ / (2017). “Características Del Buen Profesor De Modalidad Presencial Y Virtual Desde La Perspectiva De Los Estudiantes" en ( XLRSHDQ6FIHQMIF-RXLQDOr( 6-, 13(13), p. 78.

GARCÍA-PEÑALVO, F. J. (2020). "Modelo de referencia para la enseñanza no presencial en universidades presenciales" en \&DP SXV9 ILDDON, I(9), pp. 41-56.

GARCÍA-PLANAS, M. Y TABERNA TORRES, J. (2020). "The transition from the classroom to nonclassroom teaching at the UPC during the COVID-19 pandemic." en , QULQDURQDO-RXLQDORLC GXFDMRQDO 5HMDFKLDQG, QQRIDURQ 15, pp. 177-187.

MARTÍNEZ RODRÍGUEZ, F. Y GONZÁLEZ MARTÍNEZ, J. (2018). “Experiencias de los docentes pertenecientes a las facultades de ingeniería al implementar las tecnologías de la información y la comunicación (TIC) en sus prácticas educativas presenciales" en 5HILWD, QUAQDFIRQDO7FFQRQRJTDVHQDD ( GXFDFlyQ 5(1), pp. 1-13.

VITE CEVALLOS, H. (2020). "Estrategias tecnológicas y metodológicas para el desarrollo de clases online en instituciones educativas" en 5HILDD\&RQDOR, 16(75), pp. 259-265.

\section{Artículos en congresos y jornadas}

COTANO, J. B. (2005). “Las TICs en la docencia universitaria”, pp. 6-11.

(cc) EY-NC-ND 2021, Universitat Politècnica de València

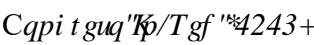


GONZÁLEZ, H. Y SERRANO, D. (2017). "Diseño y uso de materiales para la docencia presencial y

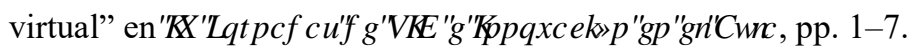

Otras fuentes consultadas

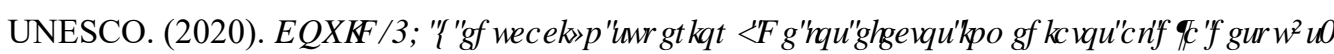

